\title{
LIFTING SEMIFREE ACTIONS
}

\author{
R. LASHOF
}

\begin{abstract}
Necessary and sufficient conditions on the structure of a bundle are given, for lifting a semifree compact Lie group acting on the base to the total space.
\end{abstract}

Let $X$ be a $G$-space, $G$ a compact Lie group, and let $p: E \rightarrow X$ be a fibre bundle. We consider the problem of lifting the action of $G$ to the total space. If $X$ is a free $G$-space, it is well known that the action lifts if and only if the bundle pulls back from a bundle over the orbit space $\bar{X}$. For general $G$-spaces the problem is quite complicated, and although we give an obstruction theory approach in [2], [3], it seems worthwhile to give an explicit solution (Theorems $\mathbf{A}, \mathbf{A}^{\prime}$ of $\$ 2$ ) in the important semifree case. If $X$ is semifree with fixed point set $F$, then $G$ acts freely on $X^{0}=X-F$ and we can apply the above criterion to $E \mid X^{0}$. Likewise it is easy to describe the $G$-actions on $E \mid F$. Thus it remains to fit these two descriptions together over a neighborhood of $F$. For this we will use the theory and classification of equivariant bundles [1], [4].

Indeed, returning to the general lifting problem, note that if a lift exists then $G$ acts on $E$ through bundle automorphisms; and if say the fibre is locally compact we can give $(E, p)$ a structure group $A$ such that $G$ acts on $E$ through $A$-bundle automorphisms. In any case, if we assume $(E, p)$ has a structure group $A$ and we consider lifts of the $G$-action to $A$-bundle automorphisms, then lifts to $E$ are equivalent to lifts to the associated principal $A$-bundle.

An $A$-bundle with $G$ acting through $A$-bundle automorphisms is called an equivariant or $G-A$ bundle if it satisfies the equivariant covering homotopy property. This requires an equivariant local triviality condition [1], [4]; but if $A$ is a closed subgroup of $\mathrm{GL}(n, R)$ for some $n$, this is automatically the case (ibid).

In [2] and [4], we have generalized Wasserman's classifying space for equivariant vector bundles to numerable equivariant $A$-bundles, $A$ arbitrary. The classifying space $B_{G} A$ is in particular a classifying space for $A$-bundles, but in addition $B_{G} A$ is a $G$-space and $G-A$ equivalence classes of $G-A$ bundles correspond bijectively with $\left[X, B_{G} A\right]_{G}$, the equivariant homotopy classes of equivariant maps of $X$ to $B_{G} A$. That is, the pull back of a $G-A$ bundle by an equivariant map is again a $G-A$ bundle, and $B_{G} A$ is the base space of a universal $G-A$ bundle. Thus if $f$ : $X \rightarrow B_{G} A$ is a classifying map for an $A$-bundle $E$ over $X$, the $G$-action on $X$ lifts to a $G-A$ structure on $E$ if and only if $f$ is homotopic to an equivariant map.

We recall the characterization of a universal $G-A$ bundle [4], and discuss some of its properties:

Received by the editors August 21, 1979.

1980 Mathematics Subject Classification. Primary 57S15, 55R10, 55R15.

(C) 1980 American Mathematical Society $0002-9939 / 80 / 0000-0430 / \$ 02.25$ 
A $G-A$ bundle $p: E \rightarrow X$ is universal if and only if for each closed subgroup $H$ of $G$ and homomorphism $\rho: H \rightarrow A$,

(1) $E$ has a fixed point under the $H$-action $z \rightarrow h z \rho(h)^{-1}, h \in H, z \in E$.

(2) For any fixed point $z_{0} \in E$ under the above action, $E$ is $H$ contractible to $z_{0}$.

A universal $G-A$ bundle will be denoted $\pi_{G} A: E_{G} A \rightarrow B_{G} A$. First note that $x \in B_{G} A$ is fixed under $H$ if and only if for any $z \in \pi^{-1}(x), h z=z \rho_{z}(h), \rho_{z}$ : $H \rightarrow Z$ a homomorphism. Further, $\rho_{z a}=a^{-1} \rho_{z} a, a \in A$. Thus each $x \in\left(B_{G} A\right)^{H}$ can be assigned a well-defined $A$-equivalence class $(\rho)$ of homomorphisms $\rho$ : $H \rightarrow A$. Now $x \in(\rho)$ if and only if there is a $z \in \pi^{-1}(x)$ such that $h z \rho(h)^{-1}=z$. By the above characterization, the fixed point set $F^{\rho}$ of $E$ under this action is contractible. Further, if $z \in F^{\rho}, z a \in F^{\rho}$ if and only if $a \in A^{\rho}=$ centralizer of $\rho(H)$ in $A$. Thus $F^{\rho}$ is an $A^{\rho}$-subbundle and indeed a universal $A^{\rho}$-bundle, since it is contractible. Thus $\pi\left(F^{\rho}\right)=B\left(A^{\rho}\right)$ and $\left(B_{G} A\right)^{H} \simeq \amalg_{(\rho)} B\left(A^{\rho}\right)$; i.e., one $\rho$ from each $A$ equivalence class. Write $B^{(\rho)}=\pi\left(F^{\rho}\right)$ and $E^{(\rho)}=\pi^{-1}\left(B^{(\rho)}\right)$. Then $E^{(\rho)}=$ $E\left(A^{\rho}\right) \times_{A^{\rho}} A$, as an $A$-bundle.

1. Bundles over a free $G$-space. If $X$ is a free $G$-space and $\bar{X}=X / G$, we have the well-known

LEMMA 1.1. There is a bijective correspondence between $G-A$ equivalence classes of $G-A$ bundles over $X$ and $A$ equivalence classes of $A$-bundles over $\bar{X}$.

In fact, if $P$ is a $G-A$ bundle over $X, \bar{P}=P / G$ is the corresponding $A$-bundle over $\bar{X}$. If $q: X \rightarrow \bar{X}$ is the quotient map and $Q$ is an $A$-bundle over $\bar{X}$ considered as a $G-A$ bundle with trivial $G$-action, then the induced bundle $q^{*} Q$ has a natural $G-A$ structure since $q$ is equivariant. These constructions preserve equivalence classes and are inverse to each other.

Now suppose we start with a $G-A$ bundle $Q$ over the trivial $G$-space $\bar{X}$ but with nontrivial $G$-action on $Q$. Then $q^{*} Q$ is again a $G-A$ bundle over $X$ but in general $\overline{q^{*} Q}$ is not $A$-equivalent to $Q$. To see just how $\overline{q^{*} Q}$ is related to $Q$, first note that $Q$ is classified by an equivariant map $f: \bar{X} \rightarrow B_{G} A$, and $f(\bar{X}) \subset\left(B_{G} A\right)^{G}=$ $\amalg_{i} B^{\left(\rho_{i}\right)}, \rho_{i}: G \rightarrow A$. Assuming $\bar{X}$ is connected, $f(\bar{X})$ is contained in a single component of $\left(B_{G} A\right)^{G}$, say $B^{(\rho)}, \rho: G \rightarrow A$. Since $E^{(\rho)}=E A^{\rho} \times_{A^{\rho}} A, Q=$ $Q_{0} \times_{A^{\rho}} A, Q_{0}$ an $A^{\rho}$-bundle, with $g\left[z_{0}, a\right]=\left[z_{0}, \rho(g) a\right]$. Considering $X$ as a principal $G$-bundle over $\bar{X}$ and letting $\gamma: G \times A^{\rho} \rightarrow A$ be the homomorphism $\gamma\left(g, a_{0}\right)$ $=\rho(g) a_{0}$, we can form the $\gamma$-product, $X \otimes_{\gamma} Q_{0}$, an $A$-bundle over $\bar{X}$. That is, $X \times Q_{0}$ is a $G \times A^{\rho}$ bundle over $\bar{X} \times \bar{X}$ and $X \otimes_{\gamma} Q_{0}$ is the pull back via $\Delta$ : $\bar{X} \rightarrow \bar{X} \times \bar{X}$ of the associated bundle with group $A$. Then by a simple check of definitions we get

LEMMA 1.2. $q^{*} Q \simeq q^{*}\left(X \otimes_{\gamma} Q_{0}\right)$ as $G-A$ bundles (or equivalently, $\overline{q^{*} Q} \simeq$ $X \otimes_{\gamma} Q_{0}$ as $A$-bundles).

Proof.

$q^{*} Q=q^{*} Q_{0} \times_{A^{p}} A=\left\{\left[\left(x, z_{0}\right), a\right] \mid \bar{x}=p\left(z_{0}\right)\right.$ and $\left.\left[\left(x, z_{0} a_{0}\right), a\right]=\left[\left(x, z_{0}\right), a_{0} a\right]\right\}$ with $G$-action, $g\left[\left(x, z_{0}\right), a\right]=\left[\left(g x, z_{0}\right), \rho(g) a\right]$. 


$$
\begin{aligned}
q^{*}\left(X \otimes_{\gamma} Q_{0}\right)= & q^{*} X \otimes_{\gamma} q^{*} Q_{0} \\
= & \left\{\left[\left(x, x^{\prime}\right),\left(x^{\prime \prime}, z_{0}\right), a\right] \mid \bar{x}=\bar{x}^{\prime}=p\left(z_{0}\right)\right. \text { and } \\
& {\left.\left[\left(x, g^{-1} x^{\prime}\right),\left(x^{\prime \prime}, z_{0} a_{0}\right), a\right]=\left[\left(x, x^{\prime}\right),\left(x^{\prime \prime}, z_{0}\right), \rho(g) a_{0} a\right]\right\} }
\end{aligned}
$$

with $G$-action $g\left[\left(x, x^{\prime}\right),\left(x^{\prime \prime}, z_{0}\right), a\right]=\left[\left(g x, x^{\prime}\right),\left(g x^{\prime \prime}, z_{0}\right), a\right]$. Note that since $q^{*} X$ has a natural cross-section there is a natural injection $i_{2}: q^{*} Q_{0} \rightarrow q^{*} X \otimes_{\gamma} q^{*} Q_{0}$; namely,

$$
i_{2}\left(x, z_{0}\right)=\left[(x, x),\left(x, z_{0}\right), 1\right] .
$$

Hence we can define a unique $A$-bundle equivalence $\varphi: q^{*} Q_{0} \times{ }_{A^{p}} A \rightarrow$ $q^{*} X \otimes_{\gamma} q^{*} Q_{0}$ extending $i_{2}$; i.e.,

$$
\varphi\left[\left(x, z_{0}\right), a\right]=\left[(x, x),\left(x, z_{0}\right), a\right] .
$$

It is easy to check that $\varphi$ is well defined and a $G-A$ bundle equivalence.

ADDENDUM. There is a unique $G-A$ bundle equivalence, indeed a unique $A$-bundle equivalence $\varphi$ extending the identity map in the subspace $q^{*} Q_{0}$ :

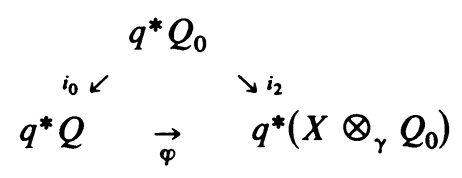

There are classifying map versions of 1.1 and 1.2:

Lemma $1.1^{\prime}$. If $X$ is a free $G$-space, $\left[X, B_{G} A\right]_{G} \simeq[\bar{X}, B A]$. Further, if we identify $B A$ with $B^{\left(\rho_{0}\right)} \subset\left(B_{G} A\right)^{G}$, then the correspondence is given by $\bar{f}: \bar{X} \rightarrow B^{\left(\rho_{0}\right)}$ going to $\bar{j}_{0} \bar{f}_{q}: X \rightarrow B^{\left(\rho_{0}\right)} \subset B_{G} A$. ( $\rho_{0}$ is the trivial homomorphism.)

Proof. As shown in [2], [5], if $X$ is a free $G$-space and $Y$ an arbitrary $G$-space, equivariant maps of $X$ to $Y$ correspond to cross-sections of the fibration $X \times{ }_{G} Y$ $\rightarrow \bar{X}$ associated to $g: X \rightarrow \bar{X}$. If $Y_{0} \subset Y^{G}$ is a deformation retract of $Y$, then $\bar{X} \times Y_{0}=X \times{ }_{G} Y_{0} \hookrightarrow X \times{ }_{G} Y$ is a f.h.e. Thus the space of equivariant maps of $X$ to $Y$ has those of the form $\bar{f} q, \bar{f}: \bar{X} \rightarrow Y_{0}$, as deformation retract. Taking $Y=B_{G} A$ and $Y_{0}=B^{\left(\rho_{0}\right)}$, the result follows.

The lemma below is equivalent to 1.2 .

LEMMA $1.2^{\prime}$.

$$
\begin{array}{cccccc}
E G \times B^{(\rho)} & \stackrel{\rho r_{2}}{\rightarrow} & B^{(\rho)} & & \\
& & & \searrow \bar{j} & \\
q \times 1 \downarrow & & & & B_{G} A \\
B G \times B^{(\rho)} & & & B^{\left(\rho_{0}\right)} & & \\
& \vec{\gamma}_{0} & & &
\end{array}
$$

commutes up to equivariant homotopy.

Proof. If we take $X=E G \times B^{(\rho)}$ and $Q=B G \times E^{(\rho)}$, then $\overline{j p r} r_{2}$ classifies $q^{*} Q$ and $\bar{j}_{0} \bar{\gamma}_{0}(q \times 1)$ classifies $q^{*}\left(X \oplus_{\gamma} Q_{0}\right)$. Thus (1.2) implies (1.2'). 
Conversely, if $\bar{f}: \bar{X} \rightarrow B^{(\rho)}$ classifies $Q_{0}$ and $\lambda: X \rightarrow E G$ is any $G$-map, then $\overline{j f} q=\overline{j p} r_{2}(\lambda, \bar{f} q)$ induces $q^{*} Q$. By $\left(1.2^{\prime}\right)$, this is equivariantly homotopic to $\bar{j}_{0} \bar{\gamma}_{0}(q \lambda, \bar{f} q)=\bar{j}_{0} \bar{\gamma}_{0}(\bar{\lambda}, \bar{f}) q$, which induces $q^{*}\left(X \otimes_{\gamma} Q_{0}\right)$. Hence (1.2') implies (1.2).

2. Bundles over a semifree $G$-space. Let $X$ be a semifree $G$-space. Let $F=\amalg_{i} F_{i}$ be the decomposition of the fixed point set into connected components. We assume $F$ is an equivariant neighborhood deformation retract. (This holds for example if $X$ is a smooth $G$-manifold or more generally a $G-\mathrm{CW}$ complex.) Let $\left\{N_{i}\right\}$ be disjoint closed invariant neighborhoods of the $F_{i}$ and $r_{i}: N_{i} \rightarrow F_{i}$ equivariant (deformation) retracts. Let $N=\amalg_{i} N_{i}$, and $N^{\prime}$ a smaller open invariant neighborhood of $F$; i.e., $\operatorname{cl}\left(N^{\prime}\right) \subset$ Int $N$. Write $X^{0}=X-N^{\prime}, N^{0}=N-N^{\prime}$ and $N_{i}^{0}=N_{i}-N^{\prime}$. Also write $\overline{X^{0}}, \overline{N^{0}}, \overline{N_{i}^{0}}$ for the orbit spaces and $q_{0}: X^{0} \rightarrow \overline{X^{0}}$, etc., for the quotient maps. Also let $\overline{\lambda_{i}^{0}}: \overline{N_{i}^{0}} \rightarrow B G$ be a classifying map for the $q_{i}^{0}: N_{i}^{0} \rightarrow \overline{N_{i}^{0}}, \lambda_{i}^{0}: N_{i}^{0} \rightarrow E G$ the covering bundle map.

Theorem A. The $G$-action on $X$ lifts to a $G-A$ structure on an $A$-bundle $E$ over $X$ if and only if the bundle has the form:

(1) $E \mid N_{i}=r_{i}^{*} P_{i} \times_{A^{\rho_{i}}} A, P_{i}$ an $A^{\rho_{i} \text {-bundle over }} F_{i}$, some $\rho_{i}: G \rightarrow A$.

(2)(a) $E \mid X^{0}=q^{*} E^{0}, \overline{E^{0}}$ an $A$-bundle over $\overline{X^{0}}$, with

(b) $\overline{E^{0}} \mid \overline{N_{i}^{0}}=N_{i}^{0} \otimes_{\gamma_{i}} \overline{r_{i}^{0} *} P_{i}, \gamma_{i}: G \times A^{\rho_{i}} \rightarrow A, \gamma_{i}(g, a)=\rho_{i}(g) a$.

(3) $E=E|N \cup E| X^{0}$, where over $N_{i}^{0}$ we identify $q^{*}\left(N_{i}^{0} \otimes_{\gamma_{i}} \overline{r_{i}^{0 *}} P_{i}\right)$ with $r_{i}^{0 *} P_{i} \times{ }_{A^{p_{i}}} A=q^{*} r_{i}^{0 *} P_{i} \times{ }_{A^{p_{i}}} A$ by the unique $A$-isomorphism extending the identity map in $q^{*} \bar{r}_{i}^{0} P_{i}$.

Proof. (a) Suppose we have a $G-A$ bundle $E$ over $X$.

(1) Since the classifying map $f: X \rightarrow B_{G} A$ sends $F$ to $\left(B_{G} A\right)^{G}$, each $F_{i}$ maps to

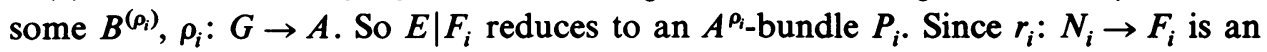
equivariant deformation retract

$$
E\left|N_{i}=r_{i}^{*} E\right| F_{i}=r_{i}^{*} P_{i} \times_{A^{p_{i}}} A=q^{*}\left(\bar{r}_{i}^{*} P_{i} \times_{A^{p_{i}}} A\right) .
$$

(2) Since $X^{0}$ is a free $G$-space, Lemma 1.1 says $E \mid X^{0}=q^{*} \overline{E^{0}}$, where $\overline{E^{0}}=$ $\left(E \mid X^{0}\right) / G$.

Since $E \mid N_{i}^{0}=q^{*}\left(\bar{r}_{i}^{0} P_{i} \times A_{A^{p_{i}}} A\right)$ we may identity $\overline{E^{0}} \overline{N_{i}^{0}}$ with $N_{i}^{0} \otimes_{\gamma_{i}} \overline{r_{i}^{0}}{ }^{*} P_{i}$ by Lemma 1.2.

By Lemma 1.2, Addendum, this means

(3) $q^{*}\left(N_{i}^{0} \otimes_{\gamma_{i}} \overline{r_{i}^{0}} * P_{i}\right)$ is identified to $q^{*} \overline{r_{i}^{0}} * P_{i} \times{ }_{A^{p_{i}}} A$ by the unique $A$-isomorphism extending the identity map on $q^{*} r_{i}^{0} * P_{i}$.

(b) Suppose we have an $A$-bundle $E$ satisfying (1), (2), (3) of Theorem A.

(1) Give $E \mid F_{i}=P_{i} \times_{A^{p_{i}}} A$ the $G$-action, $g[z, a]=\left[z, \rho_{i}(g) a\right]$. Give $E \mid N_{i}=$ $r_{i}^{*}\left(P_{i} \times A_{A^{p}} A\right)$ the induced $G$-action.

(2) Give $E \mid X^{0}$ the induced $G$-action from the trivial $G$-action on $\overline{E^{0}}$.

(3) The identification of $E \mid N$ with $E \mid X^{0}$ over $N_{i}^{0}$ is then a $G-A$ equivalence by condition (3) (see 1.2, Addendum) and thus $E=E|N \cup E| X^{0}$ has a $G-A$ structure.

Theorem A may be reformulated in terms of classifying maps. For this purpose it will be convenient to replace $X=N \cup_{{ }^{1} N^{0}} X^{0}$ by $\hat{X}=N \cup_{{ }^{1} N^{0}} N^{0} \times I \cup_{{ }^{1} N^{0}} X^{0}$ the 
space of the same $G$-homotopy type obtained by thickening $N^{0}$.

TheOREM $\mathrm{A}^{\prime}$. A map $f$ of $\hat{X}$ into $B A$ defines an $A$-bundle over $\hat{X}$ which admits a $G-A$ structure if and only if for some collection of homomorphisms $\rho_{i}: G \rightarrow A, f$ has (up to homotopy) the form:

$$
f \mid N_{i}: N_{i} \stackrel{r_{i}}{\rightarrow} F_{i} \stackrel{h_{i}}{\rightarrow} B A^{\rho_{i}} \stackrel{\bar{j}_{i}}{\rightarrow} B A,
$$

$\overline{j_{i}}$ induced by the inclusion $j_{i}: A^{\rho_{i}} \rightarrow A$.

$$
f \mid X^{0}: X^{0} \stackrel{q^{0}}{\rightarrow} \overline{X^{0}} \stackrel{\overline{f^{0}}}{\rightarrow} B A,
$$

with

$$
\overline{f^{0}} \mid \overline{N_{i}^{0}}: \overline{N_{i}^{0}} \stackrel{\left(\bar{\lambda}_{i}, h_{i}{\overline{r_{i}^{0}}}^{\rightarrow}\right)}{\rightarrow} B G \times B A^{\rho_{i}} \stackrel{\bar{\gamma}_{i}}{\rightarrow} B A,
$$

$\bar{\gamma}_{i}$ induced by $\gamma_{i}, \gamma_{i}(g, a)=\rho_{i}(g) a$.

(3) $f \mid N_{i}^{0} \times I$ is the homotopy from $\overline{f^{0}} g^{0} \mid N_{i}^{0}$ to $\overline{j_{i}} h_{i} r_{i}^{0}=\bar{j}_{i} h_{i} \bar{r}_{i}^{0} q^{0}$ derived by deforming $\bar{\lambda}_{i} q_{i}^{0}=q \lambda_{i}^{0}: N_{i}^{0} \rightarrow B G$ to a constant map.

REMARK. In (3) the map

$$
\overline{f^{0}} q^{0} \mid N_{i}^{0}: N_{i}{ }^{0} \stackrel{\left(\lambda_{i}^{0}, h_{i} r_{i}^{0}\right)}{\rightarrow} E G \times B A^{\rho_{i}} \stackrel{q \times 1}{\rightarrow} B G \times B A^{\rho^{9}} \stackrel{\overline{\gamma_{i}}}{\rightarrow} B A
$$

is deformed to $\bar{\gamma}_{0} \circ \bar{i}_{0} \circ h_{i} r_{i}^{0}, i_{0}: A^{\rho_{i}} \rightarrow G \times A^{\rho_{i}}, i_{0}(a)=(1, a)$, by the contractibility of $E G$. But $j_{i}=\gamma_{i} \circ i_{0}$.

Theorem $\mathrm{A}^{\prime}$ follows from Lemmas $1.1^{\prime}, 1.2^{\prime}$, and the structure of $B_{G} A$ described above.

EXAmple. Complex $n$-plane bundles over a semifree space $X$. Then $A=U(n)$ and any $\rho: G \rightarrow U(n)$ is equivalent to a direct sum of irreducible representations; say $\rho=n_{1} \rho_{1} \oplus \cdots \oplus n_{k} \rho_{k}$. The centralizer of $\rho$ is the subgroup $A^{\rho}=\left(U\left(n_{1}\right) \otimes I_{d_{1}}\right)$ $\oplus \cdots \oplus\left(U\left(n_{k}\right) \otimes I_{d_{k}}\right)$, where $d_{j}=\operatorname{dim} \rho_{j}$.

Thus if $F$ is a component of the fixed points of $G$ on $X$, and $E$ is a complex vector bundle over $X$ such that the action lifts, then for some $\rho: G \rightarrow U(n)$, as above

(a) $E \mid F=\left(E_{1} \times \varepsilon_{d_{i}}\right) \oplus \cdots \oplus\left(E_{k} \times \varepsilon_{d_{k}}\right)=\bigoplus_{i} d_{i} E_{i}, E_{i}$ an $n_{i}$-dimensional vector bundle.

(b) $\bar{E} \mid \bar{N}_{0}=E_{1} \otimes E_{1}^{\prime} \oplus \cdots \oplus E_{k} \otimes E_{k}^{\prime}, E_{i}^{\prime}=N_{0} \times{ }_{\rho_{i}} C^{d_{1}}, N_{0}=N-F, N$ an invariant neighborhood of $F$.

\section{BIBLIOGRAPHY}

1. E. Bierstone, The equivariant covering homotopy property for differentiable G-fibre bundles, J. Differential Geometry 8 (1973), 615-622.

2. R. Lashof, Obstructions to equivariance and lifting actions in bundles, Univ. of Virginia, mimeographed notes, 1977.

3. __ Obstructions to equivariance, Topology Conference, Aarhus, 1978.

4. R. Lashof and M. Rothenberg, G-smoothing theory, Proc. Sympos. Pure Math., vol. 32, Amer. Math. Soc., Providence, R. I., 1978, pp. 211-266.

5. C. Kosniowski, Equivariant cohomology and stable cohomotopy, Math. Ann. 210 (1974), 83-104.

Department of Mathematics, University of Chicago, Chicago, Illinois 60637 